\title{
Design of Cognitive Support for Healthcare
}

\author{
G. Lintern $^{1}$ and A. Motavalli ${ }^{2}$ \\ ${ }^{1}$ Monash University Accident Research Centre, Building 70, Monash University, Victoria 3800, Australia, \\ Email: glintern@CognitiveSystemsDesign.net \\ ${ }^{2}$ Department of Anaesthesia, The Northern Hospital, 185 Cooper St, Epping , Victoria, 3076 , Australia
}

\begin{abstract}
Healthcare work is, to a considerable extent, cognitive. Subsequently, the analysis and the design of supporting technology must be sensitive to the cognitive and adaptive demands of the work and to the cognitive strategies employed by healthcare practitioners. Despite the vital role that cognition plays in healthcare work, current technocentric design approaches for healthcare technology do not explicitly account for it, failing to observe it during analysis and thereby missing opportunities to develop supports for it during design.

Here we describe significant cognitive challenges commonly encountered by staff in high-pressure healthcare settings and observe that the common flow chart analysis used by information technology professionals does not account for any of them. In addition, we describe several design concepts that address the cognitive demands of work. These differ markedly from the rule-based decision supports or procedural interventions preferred by information technology professionals.

We argue for a paradigm shift in the design of healthcare technology and situate our argument within the broader design strategy of functional workspace design. The term workspace is preferred to interface to emphasize that the worker must remain fully immersed in the work and should not be separated from it by the technology. The term functional emphasizes that all transactions, whether they be information access, cognition, or action, carry significant meaning in relation to the work. The aim of functional workspace design is to amplify and extend the human capability to know, perceive, decide, plan, act and collaborate, by integrating system functions with the cognitive processes they need to support. The focus is on the cognitive work with an emphasis on how we might employ technological functionality to support that work.
\end{abstract}

Keywords: $\quad$ Healthcare technology, cognitive analysis, cognitive design, functional workspace design 


\section{INTRODUCTION}

Healthcare is an information rich environment. It is complex, involving many interdependent, knowledgeintensive disciplines, and is dynamic, involving knowledge that is being constantly revised, extended, and applied. Furthermore, this information environment extends beyond clinical care to include management, funding, and policy. Computerization of such an information environment would seem to offer enormous safety, performance, and economic benefits. Nevertheless, many efforts at computerizing healthcare information have led to clumsy systems that do not properly support the work as intended and that induce error (Karsh, Weinger, Abbott \& Wears, 2010). Notably, healthcare professionals are often positive about the introduction of innovative technology, only to become disenchanted when they discover that it disrupts their workflow (Lapointe \& Rivard, 2006).

In large part, the design concept and design execution for healthcare information systems remains the responsibility of information technologists whose appreciation of the scope and complexity of healthcare workas-done is limited (Nemeth, Wears, Patel, Rosen \& Cook, 2011). Attempts by healthcare professionals to influence the design are fragmented at best and often amount to little more than the generation of an incomplete list of functional requirements as developed by a small, non-representative, albeit knowledgeable group of healthcare specialists (Karsh et al, 2010). Alternatively, an expert panel of healthcare professionals may be constrained to selecting a system from those already on the market (Lapointe \& Rivard, 2006). Nothing about this process approximates a design strategy that could lead to a coherent, robust, and effective information system that would support the diverse, complex, and dynamic demands of healthcare work.

Elsewhere, we have outlined a cognitively-focused strategy aimed at designing a robust and effective work system by enhancing the cognitive processes critical to the work (Lintern \& Motavalli, submitted). Typically, those who design technological supports for healthcare work ignore the cognitive processes associated with clinical work as it is done, preferring to focus on developing systems that support clinical work as they imagine it is done (Nemeth et al, 2011). Cognitive designers focus on technological support systems such as interfaces, information-entry systems and communication systems, and on human resource issues such as team design, organizational design, staffing, selection and training. In contrast to the technology-centric design assumptions of stable, routine and knowable work processes, cognitive designers assume that healthcare work is demanding, fluid and unpredictable, being distributed and shared across a system of functionally interdependent actors and artefacts. The goal in such an environment is to establish a robust system in which the human capability to perform cognitive work is optimized.

In Lintern and Motavalli (submitted), we used case studies to illustrate the problems that emerge from designing cognitive support systems based on the pervasive techno-centric view of work practice and to demonstrate what is possible for healthcare technological support by application of a systematic cognitive design strategy.

One implication to be drawn from our argument in Lintern and Motavalli (submitted) is that healthcare technology design needs to transition through a paradigm shift that involves both the analysis of healthcare work and the design of solutions developed to support it. In this paper, we make that claim explicit. We develop our argument by reference to cognitive processes typically ignored in the development of information technology for healthcare. We accomplish this by analyzing the design implications that emerge from published studies of work processes in busy hospital settings (Nemeth, Nunnally, O’Connor et al, 2007; Nemeth et al, 2011). We further observe that the design solutions preferred by information technology professionals rely heavily on inflexible templates and standardized protocols that do not accommodate the complex and dynamic challenges posed by various health issues (Greenhalgh, Potts, Wong, Bark \& Swinglehurst, 2009; Karsh et al, 2010). Modern computer power supports diverse information formats, many of them pictorial, and diverse action capabilities, many of which are direct. The design aspect of our paradigm shift takes full advantage of those capabilities.

\section{COGNITIVE ANALYSIS AND DESIGN}

\subsection{Cognitive Issues}

Nemeth et al (2011) examined patient flow (arrival, treatment, release or transfer) through the emergency departments of two urban academic medical centers. Soon after their arrival at the emergency department, prospective patients were processed through a triage evaluation. Those with no apparent need for emergency care were encouraged to seek care elsewhere while the others were sorted into various streams depending on their triage evaluation. 
Because of limited staffing, facilities and funding, these emergency departments typically operated at or near maximum capacity, giving clinicians little opportunity to reflect within their diagnostic work on challenging issues. To cope, clinicians developed their own strategies for organizing their workflow. For example, patient loads could create a mismatch between available resources and demand for care. To help them cope with this resource-demand mismatch, clinicians sorted patients into separate queues that matched need to available care types.

Waiting times for patients could be so long that many patients left without seeing a clinician.

Within these emergency departments, coordination of care for individual patients was fragile because care was distributed among several healthcare professionals who did not always coordinate well.

Clinicians could not anticipate when test results would be returned and were not advised emergently of important abnormal results.

The procedures associated with release of patients from the emergency department concerned some clinicians. Patients who were released to home or to the street were rarely followed up after release. Nor were they contacted by the hospital to check on their health or progress. Further, there was no means of ensuring that these patients understood their diagnosis and there was no means of confirming that they understood their medication procedures or what they needed to do to manage their condition.

Finding a hospital bed for a patient who needed treatment beyond what could be provided in the emergency department could present a vexing challenge. The patient would have to be transferred within the hospital to a subspecialty service that could care for the health problem the patient was experiencing. Emergency department staff members would need permission of that service before they could admit the patient to the hospital, but this could become a difficult negotiation because services staff often resisted accepting patients from the emergency department. Furthermore, emergency department staff were impeded in their coordination with admission staff because they had different administrative tools.

The challenge of finding a suitable hospital bed for a patient who was designated for admission to a ward or an intensive care bed could sometimes result in the patient waiting in the emergency department for many hours, taking up valuable emergency-department bed space. Such a patient would be in administrative limbo; released administratively but still there physically. They were now essentially phantom patients with no official documentation. Emergency department staff developed unofficial strategies to cope, such as a binder to track the care provided to these non-patients and a dummy-bed strategy to manage admissions to hospital services.

Possibly, the most generic issue was one of poor coordination between departments, resulting in part from conflicting agendas.

\subsection{Issues for Information Technology Support}

The summary of Nemeth et al (2011) suggests several issues that might be addressed in part with a technology solution. We list those issues here. For some issues, we also list technology requirements (in parentheses) as developed by author $\mathrm{AM}^{1}$ :

- resource-demand mismatches (support stakeholder capacity to understand and to anticipate the availability, redundancy, need, and implications of (i) waiting room capacity in relation to current and anticipated patient inflow and outflow, (ii) emergency room number and types (including resuscitation, isolation, minor procedure rooms) in relation to triaged patient numbers and provisional diagnoses, and anticipated patient inflow and outflow, (iii) health practitioner numbers types and levels of expertise in relation to current and anticipated nature of workload (across hospital departments and not just the emergency department), and (iv) intra and inter-hospital bed types and numbers according to current and projected demands;

- care coordination (timely updating of the nature and severity of patient condition with guidance for subsequent care management according to test results or response to interventions);

- $\quad$ uncertainty relating to the return of test results (capacity for pathology and radiological testing, and timely communication of results);

- $\quad$ patient follow-up;

- $\quad$ patient advice and guidance;

\footnotetext{
${ }^{1}$ An anesthesiologist with 12 years' experience who has worked within a hospital emergency department as a consulting clinician for one year and continues to engage with emergency departments on a semi-regular basis.
} 
- $\quad$ location of a suitable bed for a patient who is to be admitted; and

- $\quad$ negotiation with hospital services staff in the ward or unit designated for the patient by the emergency department clinician (facilitate information transactions and negotiation between emergency department clinicians and bed managers).

\subsection{Information Technology Response}

The current approach to development and design of healthcare information systems is guided by a rational, technocratic world view (Greenhalgh, et al, 2009; Vanderhook \& Abraham, 2017) that substitutes designer judgment for clinician judgment (Karsh et al, 2010). Healthcare technologies are typically designed as rulebased decision supports or procedural interventions suited to well-ordered work environments. Problematically, these technologies do not take account of the cognitive challenges or the adaptive demands found in much healthcare work.

Typically, information technology professionals will approach this problem by developing a flowchart. Nemeth et al (2011) show a flowchart, developed within an earlier project, for the movement of patients through these emergency departments. Not one of the issues we list above is represented in that flowchart.

\subsection{Cognitive design}

Nemeth et al (2011) report that one of the difficulties faced by emergency department clinicians in coordinating with staff from the hospital services was that their information systems were incompatible. It is a basic requirement of our approach to cognitive design that the various hospital services are fully interconnected on the backbone of a common system. This is not, however, a cognitive issue and further, we assume that now, several years after the research was conducted, full interconnection on a common system is more likely.

For the issues identified, we offer the following suggestions:

- Admission and streaming; an information dashboard that helps emergency department staff anticipate consult timing and resource allocation for current emergency patients and for those in the waiting room with diagnoses and who have been assigned a triage category; which would also to help a receiving doctor advise a referring outsider to send a patient elsewhere if that were to be better for the patient.

- $\quad$ Patient waiting times; a summary displayed to patients in the waiting room showing likely wait times, to help inform their decision to stay or leave.

- Test results; an information dashboard that allows a clinician to view the workload of the testing laboratory and other pertinent details such as number of technicians on duty, as well as the position of their tests in any queue, together with an estimate of the time that the results will be available for delivery (a countdown alert), and an indication of any interim result that suggests an important abnormality.

- Orders for blood products and medications; an information dashboard for processing orders for and delivery of surgical and non-surgical intervention resources (e.g., blood products, medications), like the one developed for test results.

- Resource-demand mismatches; a workflow organization tool that helps clinicians match patient needs for care with available resources.

- $\quad$ Patient follow-up; patient contact details migrated automatically to a follow-up service that contacts patients within a specified period.

- Patient advice and guidance; following Militello, et al (2016), a one-page educational brochure for the health issue being presented, designed to help a clinician discuss with the patients their diagnosis, their medication procedures, and what they need to do to manage their condition.

- Location of a suitable bed; a shared resource-constraint space, structured in terms of patient factors (subspecialty, severity, likelihood of need for certain types of care) with drag-and-drop input from the emergency department clinician, to show a suggested determination of which specialty unit or ward the patient should go to on the basis of current provisional diagnoses, past medical history, previous admissions and diagnoses, and prior agreed criteria by the specialty units for acceptance; to be updated or overridden on the basis of test results and resource availability, together with current resource availability and constraints in that and other units or wards of interest.

Because emergency departments often operate at or near maximum capacity, it is essential that technology supports do not create additional work. They must be focused primarily on helping healthcare practitioners perform their cognitive work, particularly the work that is tactical and strategic. Time for reflection and planning is essential. Given the intensive nature of this work, cognitive supports that ease the load of routine 
work and that create opportunities for assessing, diagnosing, planning and coordinating are likely to be well received by clinicians and are likely to enhance the quality of healthcare.

\subsection{Negotiation}

Negotiations within a large hospital can involve administrators, bed managers, ambulance personnel, patients, and healthcare professionals within and outside the department, and can cover issues such as healthcare resource availability, management planning, resource allocation, care implementation, and follow-up processes. The difficulty of these negotiations can be exacerbated by conflicting agendas. The work of Nemeth et al (2011) and Nemeth et al (2007) has focused on negotiation between healthcare professionals.

The transfer of a patient from the emergency department to a specialty unit or ward as described by Nemeth et al (2011) is illustrative. That transfer is preceded by a negotiation between the emergency department clinician and the hospital services admission staff. For those of us who are not healthcare professionals (e.g., author GL), this would seem to be a straightforward negotiation in which patient need is matched to ward capabilities. Experienced clinicians (e.g., author AM) would realize that this is more than a logical problem of matching need to resource and they would appreciate that they must enter this negotiation with their best arguments and agenda in mind.

Nemeth et al (2007) describe how a call schedule can support adaptive assignment of anesthesia services in response to unscheduled contingencies. While there is a routine type and level of demand that can be anticipated for each day's procedures, there is much that cannot be scheduled in advance. An emergent case such as a liver or heart becoming available for transplant, or one or more scheduled clinicians calling in sick, will place additional scheduling demands on the system. The call schedule gives the anesthesia coordinator multiple options for covering diverse types of contingencies. The call schedule is a procedural response for hedging against uncertainty. It has been developed organically and is accepted by clinicians as the appropriate strategy to negotiate assignment for an emerging demand.

There is much written on inter-personal negotiation, and a consideration of that topic is beyond the scope of this paper, but suffice to say that negotiation between competent and dedicated professionals is an inescapable feature of healthcare. Those negotiations are often routine but are sometimes difficult and challenging and, at least for some of those involved, the outcomes can be unsatisfactory. Within healthcare, the resolution of agenda conflicts relies heavily on diplomacy and on professional enculturation (Nemeth et al, 2007).

One of the challenges we pose for healthcare information technology is to develop systems that can support negotiation. At the most basic level, those entering a negotiation should be able to access the relevant information (presented in the form that clarifies its relevance to the problem in a transparent fashion) before and as they engage in the discussion. Our suggestion above for a dashboard that would assist with location of a suitable bed for a patient would satisfy at least some of the requirement for that problem, but we imagine there is far more to negotiation than having the facts at hand, and we think of that as a capability we should explore soon. At the very least, technology designers should ensure that their healthcare support systems will complement (not preclude or disrupt) organically developed and professionally accepted negotiation strategies.

\section{FUNCTIONAL WORKSPACE DESIGN}

The design solutions that emerge from the rational model of healthcare are based on overly-simplified decisionsupport rules that disrupt clinical workflows, and on inflexible templates and standardized protocols that do not accommodate the complex and dynamic challenges posed by diverse health issues (Greenhalgh, et al, 2009; Karsh et al, 2010). In Lintern and Motavalli (submitted), we note that healthcare information technology relies on clumsy and labor-intensive features such as data-entry windows, drop-down menus, lists with check boxes, and alarms.

These design features emerge from a limited view of design; one that we would prefer to replace with seamless navigation between functional entities, drag-and-drop capabilities, voice and gesture commands, multi-sensory information systems, and numerous other design features that link naturally to human capability. Lintern, Waite and Talleur (1999) have outlined this approach as it applies to aviation, while Bennett and Flach (2011) have shown how it can be applied to a range of domains. Watson and Sanderson (2007) have explained how it can inform the design of sonic displays for anesthesiology.

In our effort to promote a paradigm shift for design of healthcare technology, we wish to transition from viewing the resulting product as an interface. Rather, we characterize it as a functional workspace. The term workspace is preferred to interface to emphasize that worker must remain fully immersed in the work and should not be separated from it by the technology. A medical doctor in an emergency department is a clinician, 
not a user. As designers, we must foreground the clinical work as we make the technology invisible (Bodker, 1991). The term functional emphasizes that all transactions, whether they be information access, cognition or action, carry significant meaning in relation to the work.

Within a functional workspace, information is structured in a manner that reflects the structure of the cognitive work. The information is thereby readily assimilated and there are natural transitions between information elements. While a functional workspace presents more information than a conventional interface, it does not overload the worker because that information is integrated across levels of abstraction and the workspace supports a natural and compatible navigation that allows the worker to converge naturally on currently important constellations of information. Although a functional workspace reduces complexity of activity, it does not do that by reducing the complexity of the information but rather by managing that complexity.

The emphasis is on meaningful information that supports functional action, versus data that must be interpreted. In most systems, we are less concerned with pieces of information than we are with constellations of information as they support cognition. For example, patient information can be presented as contextualized patterns that enable a health-care worker to assess the health status with minimal cognitive effort. The interpretation of such a display is direct and immediate. More generally, cognitive processing effort can be minimized and meaningful interaction enhanced by use of a constellation of ideas encompassing direct perception, direct manipulation, and mental models.

The vision for a functional workspace is one of a fully integrated and comprehensive information-action system that relies heavily on visualization and employs many computerized means of transforming, selecting, highlighting and associating information. It will employ icon libraries, work templates, drag-and-drop functionality, selection by mouse click, association by linking with mouse action, interrogation of concepts to bring up more detail or context, and convenient modeling tools to test outcomes of proposed actions.

Essential information will be readily accessible and presented in succinct and meaningful forms. There will be summaries of contextually relevant information and obvious signs to guide access to it. Evocative perceptual forms will be used for skill- and rule-based analysis, and the verbal information required for support of knowledge-based analysis will be summarized and highlighted so that the healthcare worker can converge readily on essential meaning as it relates to the current issue. Healthcare specialty, personal preferences, and own-patient information will be attached to security access credentials so that the workspace will reconfigure on shared computers to accommodate preferred work patterns, preferred workflows, and current patientspecific tasks for individual healthcare practitioners.

The way this can be accomplished is demonstrated in Lintern (2013).

\section{DISCUSSION}

The most pervasive concerns expressed by healthcare workers are that information is incomplete, fragmented and difficult to find. When found, information can be difficult to interpret. In addition, entry of information into a software application is often clumsy to such an extent that it distracts the healthcare professional from the essential issues of healthcare and from the patient. While some may argue that these sorts of problems are an inevitable result of technological constraints and a lack of technological sophistication among healthcare workers, we believe that an impoverished, techno-centric design perspective is the culprit.

The aim of functional workspace design is to avoid issues such as these while amplifying and extending the human capability to know, perceive, decide, plan, act and collaborate, by integrating system functions with the cognitive processes they need to support. The focus is on the cognitive work with an emphasis on how we might employ technological functionality to support that work. Functional workspace design uses formal methods of cognitive analysis and cognitive design to ensure that cognitive work is effective, efficient and robust. It has both an analysis and a design phase. Analysis is directed at understanding how domain specialists go about their work; what they are trying to achieve and what resources, strategies and processes they use to accomplish their work. Design focuses on ways of supporting the work and on possibilities for employing new technological capabilities to support work more effectively.

\section{REFERENCES}

Bennett, K.B. and Flach, J.M. (2011). Display and Interface Design: Subtle Science, Exact Art. Boca Raton, FL, CRC Press, ISBN-13: 978-1-4200-6439-1 (Ebook-PDF).

Bodker, S. (1991). Through the Interface: A Human Activity Approach to User Interface Design. Hillsdale, NJ: Lawrence Erlbaum, ISBN 0-8058-0571-0. 
Greenhalgh T., Potts H., Wong G., Bark P. and Swinglehurst D. (2009). Tensions and paradoxes in electronic patient record research: a systematic literature review using the meta-narrative method. Milbank Q, 87, 72988.

Karsh B.T., Weinger M.B., Abbott P.A. and Wears R.L. (2010). Health information technology: Fallacies and sober realities. Journal of the American Medical Informatics Association, 17(6), 617-23. DOI: 10.1136/jamia.2010.005637.

Lapointe L. and Rivard S. (2006). Getting physicians to accept new information technology: insights from case studies. Canadian Medical Association Journal. 74(11), 1573-1578. doi:10.1503/cmaj.050281.

Lintern G. (2013). Joker One: A Tutorial in Cognitive Work Analysis. Melbourne, Australia: Cognitive Systems Design. Retrieved August 1, 2013, from http://www.cognitivesystemsdesign.net/Downloads/Cognitive Work Analysis Joker One Tutorial.pdf.

Lintern, G. and Motavalli, A. (submitted). Healthcare Information Systems: The Cognitive Challenge. BMC Medical Informatics and Decision Making.

Lintern, G., Waite, T. and Talleur, D. A. (1999). Functional interface design for the modern aircraft cockpit. The International Journal of Aviation Psychology, 9, 225-240.

Militello L.G., Saleem J.J., Borders M.R., et al. (2016). Designing Colorectal Cancer Screening Decision Support: A Cognitive Engineering Enterprise. Journal of Cognitive Engineering and Decision Making 10: 7490.

Nemeth C, Nunnally M, O’Connor M, et al. (2007). Regularly irregular: How groups reconcile cross-cutting agendas in healthcare. In: Nemeth C, ed. Second special issue on large scale coordination. Cogn Technol Work, 9, 139-148.

Nemeth C., Wears R.L., Patel S., Rosen G. and Cook R. (2011). Resilience is not control: healthcare, crisis management, and ICT. Cogn Technol Work. 13(3), 189-202.

Vanderhook S. and Abraham J. (2017). Unintended Consequences of EHR Systems: A Narrative Review. Proceedings of the 2017 International Symposium on Human Factors and Ergonomics in Health Care.

Watson M.O. and Sanderson P.M. (2007). Designing for Attention with Sound: Challenges and Extensions to Ecological Interface Design. Human Factors, 49 (2), 331-346. 